\title{
A Cholesterol Primer for Health Professionals
}

\author{
Rodney G. Bowden ${ }^{1}$, Stuart Hebert ${ }^{1}$, Ronald Wilson ${ }^{2}$, Mindy Gentile ${ }^{2}$, Beth A. Lanning ${ }^{1}$ \\ ${ }^{1}$ Baylor University \\ ${ }^{2}$ Central Texas Nephrology Associates
}

\begin{abstract}
As research continues in the field of coronary artery disease, more information is revealed about various etiological factors. Emerging lipoprotein risk factors have been identified and are now starting to surface as instrumental in the cause and prevention of coronary artery disease. In order to conduct comprehensive cholesterol screening programs and counseling sessions a health professional must have a thorough understanding of lipid metabolism. Recent changes in cholesterol guidelines make it necessary to have a review that addresses the specifics of lipid management. A health professional needs an appropriate knowledge base to be able to understand a major coronary artery disease risk factor and thereby more effectively educate the public about lipid management and coronary artery disease risk reduction. Therefore, the purpose of this article is to review the role of cholesterol in both normal physiological functioning and disease causation and to examine the research concerning new emerging cholesterol risk factors.
\end{abstract}

(C) 2006 Californian Journal of Health Promotion. All rights reserved.

Keywords: Cholesterol, coronary heart disease, cholesterol subfractions, cholesterol concentration

\section{Cholesterol Screening and the Health Professional}

Recommendations by the National Cholesterol Education Program (NCEP) suggest that all Americans over the age of twenty should have their cholesterol concentrations measured (National Heart, Lung, and Blood Institute [NHLBI], 2003). An estimated $70.8 \%$ of the US population twenty years and older had participated in cholesterol screening at least once by the year 2000. This is a substantial increase from the mid-1980s when $35 \%$ had been screened at least once (Brown, Giles, Greenlund, \& Croft, 2001). A number of health education programs have an emphasis on cholesterol screening followed by counseling with a health professional. Through these screenings health professionals can have a significant impact on cardiovascular disease outcomes by being involved in primary and secondary prevention, raising awareness, and successfully referring participants to physicians for further testing (Bowden, Kingery, \& Brizzolara, 1999; Muratova, Islam, Demerath, Minor, \& Neal 2001). In order to conduct comprehensive cholesterol screening programs and counseling sessions, health professionals must have a thorough understanding of coronary artery disease (CAD) risk factors which includes lipid metabolism (Ostwald, Weiss-Farnan, \& Monson, 1990). Since the most important aspect of cholesterol screening is the action the participants take after receiving their screening results (Garber \& Browner, 1997), having accurate and up-to-date information on the role of cholesterol in CAD enables health professionals to effectively develop and implement prevention programs, educate the public, and make referrals (Sullivan, 2002). Recent changes in cholesterol guidelines make it necessary to have a review that addresses the specifics of lipid management and CAD prevention. Therefore, the purpose of this paper is to review the role of cholesterol in both normal physiological functioning and disease causation and to examine the latest research concerning the new emerging cholesterol risk factors of CAD. 


\section{Overview}

According to the American Heart Association [AHA] (2002), more Americans die from CAD every year than the next five leading causes of death combined. One in every 2.5 deaths in the year 2000 was from heart disease (KohlmanTrigoboff, 2005). Though there has been a decrease in mortality rate in the US, CAD has become a leading cause of global mortality, accounting for almost 17 million deaths annually with nearly $80 \%$ of mortality and disease burden occurring in developing countries (Smith, Jackson, Pearson, Fuster, Yusuf, \& Faergeman, et al., 2004).

The etiology of CAD is multi-factorial, involving numerous factors including genetics, diet, and environment with several risk factors significantly increasing an individual's susceptibility to the disease. These risk factors include cigarette smoking, obesity, sedentary lifestyle, dietary habits, homocysteine, high blood pressure, high blood cholesterol and others. However, much of the research into $\mathrm{CAD}$, which has being quite extensive and spanning a number of decades, has focused on the general relationship between plasma lipids and CAD (Gotto, 1997; Kannel, Castelli, Gordon, \& McNamara, 1971; McGee, Reed, Stemmerman, Rhoads, Yano, \& Feinlab, 1985; NCEP, 2002; NIH, 1989a;). Researchers have suggested that approximately twenty-five percent of the adult population ages twenty and older has blood cholesterol levels that are considered high (National Institutes of Health [NIH], 1989b). In addition, researchers have demonstrated that a total cholesterol level in the "high" category (>200 mg/dL) accompanied with high blood pressure $(>130 / 85)$ increases an individual's risk of coronary heart disease by a factor of six (NIH, 1989b). Therefore, establishing specific guidelines for cholesterol levels is both important and necessary to enhance the health of individuals.

Lipoprotein metabolism is a process that is not completely understood with fragmentary findings (Tulenka \& Sumner, 2002). Attempting to have a clearer understanding of the relationship between cholesterol levels and CAD, individuals who have abnormal lipid levels can make the lifestyle changes necessary to reduce the risk of $\mathrm{CAD}$ and its associated complications. Similarly, adequately informed health professionals are better able to educate the public about cholesterol and heart disease and more equipped to implement effective health intervention programs.

\section{Cholesterol and Cardiovascular Disease Etiology}

Understanding the pathophysiology of CAD in population studies underlie the vital role of cholesterol metabolism. Protective mechanisms of the endothelium are evident in reverse cholesterol transport performed by high-density lipoprotein (HDL) and conversely low-density lipoprotein (LDL), specifically small, dense LDL, may penetrate the subendothelial space if concentrations are high in the plasma. Penetration of the endothelial space can cause acute and chronic endothelial damage, leading to CAD. Because movement into the arterial wall is likely driven by diffusion, hypercholesterolemia increases the infiltration of cholesterol into the endothelial space (Bowden, 2001; Wada \& Karino, 1999). In response to this accumulation of cholesterol, macrophages respond to inflammatory markers from inflammatory cells, cytokines, growth factors and cellular responses (Sullivan, 2002) and absorb the cholesterol resulting in the formation of foam cells. Formations of foam cells are critical in the development of plaque in the endothelium (Ockene \& Ockene, 1992). As the $\mathrm{CAD}$ progresses, lesions may begin to cause chronic injury to the endothelium. This process results in a positive-feedback cycle due to cytokine release that sends even more macrophages to the area, resulting in more foam cells, and eventually results in stenosis and occlusion of blood flow. Fatty streaks are first evident in this disease process followed by fibrous plaques that can develop necrotic cores which develop fissures leading to plague rupture. Hyperlipidemic concentrations also increase platelet aggregability, which attenuate the severity of the thrombotic process (Sullivan, 2002). Therefore, cholesterol metabolism plays a significant role in the development of plaque, stenosis, and eventually, myocardial infarction. 


\section{Total Cholesterol}

Historically, total cholesterol concentration was used to assess an individual's risk of CAD (Bowden \& Kingery, 2004). Because cholesterol contributes to the buildup of atherosclerotic plaques, an individual's blood cholesterol concentration could be a way to measure risk for heart disease. Clinical studies are consistent in supporting the projection that for serum cholesterol levels in the $250-300 \mathrm{mg} / \mathrm{dl}$ range, each $1 \%$ reduction in serum cholesterol level reduces CAD rates by approximately $2 \%(\mathrm{NIH}$, 1989a). However, the degree of stenosis and CAD varies between individuals with the same total cholesterol and other lipid levels (Bowden, Kingery, Rust, 2004, Kmietowicz, 1998; Telenko \& Sumner, 2002). Total cholesterol tends to reflect average dietary habits that affect LDL, and can reasonably provide an assessment of CVD risk between participants. Yet, the differences in risk between individuals can be strongly influenced by many additional factors. Therefore the measurement of total cholesterol alone cannot adequately reflect individual risk of CAD (NIH, 2002) and should rarely be used as the sole lipid measure in cholesterol screenings. Other studies have also demonstrated the process of heart disease to consist of many factors that are independent of total cholesterol (Katerndahl \& Lawler, 1999). These other risk factors fall into two three broad categories, consisting of blood markers, behavior, and biology. New blood tests that identify increased cardiovascular risk include various subfractions of cholesterol. Many of these new markers relate to the physiological functions of cholesterol and the interaction between these markers and the cholesterol in the periphery.

The generally accepted ranges for total cholesterol levels (NIH, 2002) consist of desirable $(<200 \mathrm{mg} / \mathrm{dL})$, borderline high $(200$ $239 \mathrm{mg} / \mathrm{dL})$, and high $(\geq 240 \mathrm{mg} / \mathrm{dL})$. If a patient's cholesterol level is in the high category, a LDL cholesterol measure should be performed. If the patient is in the borderline high range, another total cholesterol measurement should be taken within eight weeks and the average of the two readings used to guide future decisions (NIH, 2002).

\section{Cholesterol Subfractions}

LDL cholesterol accounts for $60-75 \%$ of the total serum cholesterol and is the terminal end of in the pathway of lipoprotein metabolism called cholesterol transport. Numerous epidemiological, physiological, and animal models have linked high LDL levels to CAD (American Heart Association, 2004; Assman, Cullen \& Schulte, 1998; NIH, 1989a; Smith et al., 2004; Stone, 2005). High levels of LDL cholesterol are able to penetrate the porous endothelium of arteries and begin to accumulate if plasma concentrations are abnormal. This natural plaque is eventually converted to unstable plaque increasing the likelihood of rupture and possible thrombosis (NIH, 2002). Accordingly, the greatest absolute diminution of risk can be achieved by the reduction of LDL which may directly lower platelet aggregation, vascular reactivity, and lower cytokine release leading to a further reduction in risk for myocardial infarction (Sullivan, 2002). In fact, when elevated LDL levels are combined with comorbidity factors of smoking and hypertension, this complex explains over $90 \%$ of myocardial infarction cases occurring in middle age (Wilhelmsen, 1997). The landmark INTERHEART data suggests that $90 \%$ of risk comes from combination of abnormal levels of apolipoproteins found in LDL and smoking. LDL contains ApoB-100 which has been linked to atherogenesis (Yusef, Hawken, Ounpuu, Dans, Avesum, Lanas et al., 2004). Finally, it should be noted that although LDL lowering therapy is believe to offer the greatest benefit for CAD risk reduction, LDL alone is insufficient to predict CAD incidence and risk stratification. The best risk prediction strategy requires measurement of other cholesterol components and particle size and concentration (Wald, Law, Watt, Wu et al., 1994).

LDL ranges in size from the largest and least dense (LDL1), intermediate density and size (LDL2) to the smallest and most dense (LDL3). The ATP-III report states that small LDL particles are formed in large part, although not exclusively, as a response to elevation of triglycerides via the production of very-low density lipoproteins (VLDL) and specifically VLDL1 (Malloy \& Kane, 2001; NIH, 2002). 
The presence of small, dense LDL particles is associated with more than a three-fold increase in the risk of CAD and is independent of LDL levels (Austin, Breslow, Hennekens, Buring, Willett, \& Kraus, 1988). Tulenka \& Sumner (2002) further suggest that not all LDL particles are the same and that variations in disease outcomes may by attributable to differences in particle size and number even when LDL levels are the same between patients. The authors of the Physicians Health Study demonstrated that each decrease of eight angstroms in LDL peak particle size was associated with a significant $38 \%$ increase in the seven-year risk of myocardial infarction after adjustment for age and smoking status (Lemarche, Lemieux, \& Depres, 1999).

The correlation between particle size and CAD may exist because of the physiological properties of smaller particles. Researchers suggests smaller and denser LDL particles are more susceptible to in vitro oxidation and have been shown to be degraded less rapidly (Hsueh \& Law, 1998). In addition, smaller particles diffuse more easily into the sub-endothelial space in the periphery. A stronger diffusion gradient would push more particles into the arterial wall, attract more macrophages, and develop more foam cells.

Using gel electrophoresis, previous studies have computed and investigated both LDL peak particle size and the mean LDL particle size (Hsueh \& Law, 1998). Mean LDL particle size is determined by computing the relative abundance of each of the LDL subclasses within one individual through a densitometric scan (Hsueh \& Law, 1998; Lemarche et al., 1999). The results of these studies have led to the development of two different categories of LDL classification that rely on both peak particle size and LDL subclass distribution (Tulenko \& Sumner, 2002). These two designations are Phenotype A and Phenotype B. Phenotype A consists of a predominance of LDL particles of $>25.5$ nanometers and Phenotype B is defined as the predominance of small LDL particles with diameters $<25.5$ nanometers. (Lemarche et al., 1999). Researchers continue to investigate any relationship between Phenotype B and CAD and future findings may include either mean or peak particle size recommendations.

However, Cromwell and Otvos (2004) believe it is not clear that small LDL particles are more atherogenic than large ones simply because individuals with small LDL particles also have a higher LDL particle number. The authors further state that LDL particle number measured by nuclear magnetic resonance has consistently been shown to be a strong, independent predictor of CAD. In other words, small dense particles may have been found to be more atherogenic due to a higher number of particles that are typically associated with small dense particles. Also, the combination of the two (high particle number, and small dense particles) may place individuals at more risk than either risk factor alone.

Previous studies have identified a LDL cholesterol "disconnect" between LDL concentration and the number or size of LDL particles among patients with low levels of LDL cholesterol (Otvos, Jeyarajah, \& Cromwell, 2002). The term disconnect suggests a differing risk profile depending on the type of LDL cholesterol measure that is used. Typically many individuals who are considered to have normal levels of LDL cholesterol will screen abnormal using phenotype designation. This difference, or disconnect, may help to explain why myocardial infarction can occur in some people who have normal cholesterol and/or LDL levels. Furthermore, since cholesterol is carried via lipoproteins within the blood in spherical particles, between any two individuals there can be tremendous differences in both the number, size and composition of these particles (Garvey 2003; Tulenko \& Sumner, 2002). The implication of this disconnect is that CAD risk between two patients with identical LDL particle number and particle size would be the same, despite differing LDL concentration values (Garvey, 2003; Otvos et al., 2002; Tulenko \& Sumner, 2002).

The ATP-III (NCEP) report establishes the following ranges for LDL cholesterol levels: optimal $(<100 \mathrm{mg} / \mathrm{dL})$, near optimal/above optimal (100-129 mg/dL), borderline high (130- 
$159 \mathrm{mg} / \mathrm{dL})$, high (160-189 $\mathrm{mg} / \mathrm{dL})$, and very high $(\geq 190 \mathrm{mg} / \mathrm{dL})(\mathrm{NIH}, 2002)$. When risk is very high (two or more additional risk factors of existing heart disease), an LDL goal of $<70$ $\mathrm{mg} / \mathrm{dL}$ is a therapeutic option, but lifestyle changes should still be pursued. This therapeutic option extends also to patients at very high risk who have a baseline LDL $<100 \mathrm{mg} / \mathrm{dL}$ (Grundy et al., 2004).

The metabolic balance of lipoproteins which is both vital and dangerous also uses reverse cholesterol transport to lower cholesterol in the periphery (Trigatti, 2005). HDL is synthesized by intestinal mucosal cells and the liver. It contains a small amount of phospholipids and ApoA1 (Tulenko \& Sumner, 2002). Research has consistently identified an inverse relationship between HDL levels and CAD incidence. The mechanism for this relationship is still unclear, leading some researchers to suggest that low HDL levels are simply a marker for other lipid abnormalities. While the role of decreased HDL levels in atherosclerosis is still vague, it is considered an independent risk factor for CAD (NIH, 2002). It also has been identified as the greatest predictor, along with ApoA1 as the most important risk factor in patients with existing CAD (Bolibar, von Eckardstein, Assman, \&Thompson, 2000; Devroey, 2004). HDL absorbs cholesterol in peripheral cells which enter the core of the cell through the action of lecithin-cholesterol acyltransferase. Inclusion of HDL in risk assessment can greatly enhance risk stratification (Kannel \& Wilson, 1992).

Research scientists have also demonstrated that HDL has at least three distinct subclasses based on particle size. Different subclasses include nascent HDL, HDL2, and HDL3 with nascent HDL being the smaller and more dense followed by HDL3 and HDL2. One study found gender differences were most pronounced for large HDL, with women having a twofold higher (8 vs. 4 micromole/L) concentration of large HDL particles than men. Additionally, the observed differences in males and females large HDL particle size also decreased with age (Freedman et al., 2004). The authors of a similar study found that the antioxidative activity of large
HDL was significantly higher than that of small HDL (Kontush, Chantepie, \& Chapman, 2003). Numerous small studies suggest greater predictive power for each of the HDL components including the observation that large HDL particles are more cardioprotective. All subclasses of HDL have been demonstrated to have a role in reverse cholesterol transport, but HDL2 seems to have the most protective effect, with recent evidence suggesting that HDL 3 may play a role in LDL oxidation that is just as vital (Yoshikawa, Sakuma, Hibino, Sato, \& Fujinami, 1997). Finally HDL seems to have an antioxidant, anti-inflammatory, anti-adhesive, anti-aggregatory, and profibinolytic effect that aids in the control of CAD beyond reverse cholesterol transport mechanisms (Tulenko \& Sumner, 2002).

The ATP-III recommended ranges for HDL are low $(<40 \mathrm{mg} / \mathrm{dL})$ and high $(>60 \mathrm{mg} / \mathrm{dL})$. This is a significant change as previous reports also set recommended levels for HDL, but the low designation was set at less than $35 \mathrm{mg} / \mathrm{dL}(\mathrm{NIH}$, 2002). Additionally, the third report has removed specific HDL levels for men and women, and made one recommendation of greater than $50 \mathrm{mg} / \mathrm{dL}$.

Another subclass of lipoprotein is VLDL which can be divided into VLDL1 (large and less dense), VLDL2 (smaller and more dense), and VLDL3 (smallest and most dense). Hypertriglyceridemia is associated with an excess of VLDL1 while hypercholesterolemia is associated with excess VLDL2. VLDL is triglyceride rich and contains C-II, ApoE, and ApoB-100. Lipoprotein lipase reduces the size of VLDL through the release of triglyceride creating a smaller, dense and more cholesterol rich lipoprotein. About two-thirds of VLDL passes down the lipoprotein metabolism cascade terminating as LDL (Tulenka \& Sumner, 2002). VLDL1 is a key component is what has been called the atherogenic lipoprotein profile, which when combined with small dense LDL, and low HDL, it is theorized to be a significant lipid risk factor for CAD (Austin et al., 1988). Most triglycerides are consumed from food, but during times of decreased caloric intake, the liver produces triglyceride endogenously 
(Kwiterovich, 1989). The ATP-III reports that VLDL levels should be less than $31 \mathrm{mg} / \mathrm{dL}$.

Triglycerides are a primary source of energy and their levels in the periphery vary significantly. Historically, it has been understood that high VLDL and triglyceride levels were the result of elevated total cholesterol and lower levels of HDL cholesterol (Ginsberg, 1999; Tulenk \& Sumner, 2002), but recent studies have shifted elevated triglyceride levels from an association with CAD to an independent predictor of the disease (Cullen 2000; Ginsberg, 1999; NIH, 2002; Malloy \& Kane, 2001;). Furthermore, this independent relationship suggests some triglyceride-rich lipoproteins are atherogenic (Cullen 2000; NIH, 2002), meaning VLDL levels may prove to be a significant risk factor in the future. With hypertriglyceridemia, triglycerides are transferred from VLDL and chylomicrons (cholesterol molecules formed from dietary substrates) to LDL, leading primarily to small dense LDL particles and more CAD (Tulenko \& Sumner, 2002).

Triglycerides should be measured after fasting as non-fasting triglyceride and other postprandial measurements are difficult to homogenize and arduous to perform (Sullivan, 2002). Finally, the authors of the ATP-III report set the classification of triglyceride in the following categories: Normal $(<150 \mathrm{mg} / \mathrm{dL})$, borderline high (150-199 mg/dL), high (200$499 \mathrm{mg} / \mathrm{dL})$, and very high $(\geq 500 \mathrm{mg} / \mathrm{dL})$.

Chylomicrons are very similar in their structure to VLDL, but are released by the intestinal mucosa cells directly after consuming fat (Tulenka \& Sumner, 2002). They are less dense due to their large size $(100-500 \mathrm{~nm})$ and the amount of triglyceride that is transported in them. Chylomicrons are found in the blood and lymphatic fluid where they serve to transport fat from its port of entry in the intestine to the liver and to adipose tissue. They travel via the lymphatic system and their large size renders penetration of the endothelium improbable. Though chylomicrons are large and rich in triglyceride, they contain only a relatively small amount of protein (Hertz, 1999; Schumaker \&, Lambertas, 1992). Once chylomicrons enter the blood. they acquire ApoE and ApoC-II. They gradually reduce in size by lipoprotein lipase which removes free-fatty acids from the triglyceride pool in the cell. Chylomicron remnants are reassembled with endogenous triglyceride and cholesterol esters to form VLDLs (Tulenka \& Sumner, 2002). Partially degraded chylomicrons, called chylomicron remnants, probably carry some atherogenic potential (NIH, 2002). The ATP-III does not report guidelines for chylomicron levels.

Recently, investigators from the INTERHEART study have demonstrated abnormal lipid levels, when combined with smoking, provide over $90 \%$ of the risk associated with CAD (Yusef et al., 2004) and can be generalized globally. The authors of the study suggest that the ApoB/ApoA1 ratio was the most important risk factor for $\mathrm{CAD}$. Previous research suggests that ApoB/ApoA1 has not warranted as much attention of other subfractions of cholesterol and therefore needs further study (Sullivan, 2002). The relative lack of familiarity among professionals regarding the importance of ApoB and ApoA1 levels has been a primary cause of ApoB measurement not prevailing over cholesterol levels as the basis for treatment guidelines. Finally, Sullivan (2002) suggests the stage has not been reached where ApoA1 levels can supersede HDL levels as the basis for treatment guidelines (Sullivan, 2002). INTERHEART is a landmark study that will likely reveal a greater role of the ApoB/ApoA1 ratio in the progression of CAD.

\section{Conclusions}

As research continues in the field of CAD, more information is revealed about the etiology of the disease. New emerging risk factors have been identified and are now starting to surface as instrumental in the cause and prevention of CAD. The changes between each of the NCEP reports show an increased understanding into the nature of lipid interactions. Each report represents another step forward in unraveling the mechanisms behind atherosclerosis and CAD. Evidence suggests that LDL particle number and size may play an important role in disease progression. Additionally, triglyceride and VLDL have been identified as independent risk 
factors for CAD. Finally, large HDL, specifically HDL2 and possibly HDL3 have been identified as important players in the reverse cholesterol transport.

Health professionals need to have a thorough understanding of cholesterol and lipid management. Understanding risk factors such as cholesterol and being able to explain them in layman's terms to clients, patients, and program participants is a necessary skill of health professionals. A very common approach by health professionals is a health fair involving some level of lipid screening and educational follow-up. By understanding the complex nature of CAD and lipid management, health professionals will be more effective in explaining the disease risk of participants, clients, and patients but will also understand the most appropriate time for physician referral and/or follow-up screening.

\section{References}

American Heart Association. (2004). Heart disease and stroke statistics: 2004 update. Dallas, TX: American Heart Association.

American Heart Association. (2002). Heart disease and stroke statistics-2003 update. Dallas, TX: American Heart Association.

Assman, G., Cullen, P., \& Schulte, H. (1998). The Munster heart study (PROCAM): Results of follow-up at 8 years. European Heart Journal, 19 (Suppl A), A2-A11.

Austin, M., Breslow, J., Hennekens, C., Buring, J. E., Willett, W. C., \& Kraus, R. M. (1988). Low-density lipoprotein subclass patterns and risk of myocardial infarction. Journal of the American Medical Association, 260, 1917-1921.

Bolibar, I., von Eckardstein, A., Assman, G., Thompson, S. (2000). Short-term prognostic value of lipid measurements for coronary events in patients with angina pectoris. Journal of Thrombosis and Haemostasis, 84, 955-961.

Bowden, R. G. (2001). Comparison of participants and nonparticipants in a worksite cholesterol screening program. International Electronic Journal of Health Education, 4, 100-104.

Bowden, R. G., \& Kingery, P. M. (2004). Significant predictors of elevated cholesterol in a worksite screening program. Journal of Academic Inquiries, 1(1), 2-13.

Bowden, R. G., Kingery, P., \& Brizzolara, J. (1999). Prediction accuracy and contrast of three dichotomous cholesterol measures. Journal of Health Education, September/October, 30(5), 284289.

Bowden, R. G., Kingery, P. M., \& Rust, D. (2004). Assessing risk using different cholesterol measures. American Journal of Public Health, 118(3), 225-229.

Brown, D.W., Giles, W. H., Greenlund, K. J., \& Croft, J. B. (2001). Disparities in cholesterol screening: Falling short of a national health objective. Preventive Medicine, 33, 517-522.

Cromwell, W. C., \& Otvos, J. D. (2004). Low-density lipoprotein particle number and risk for cardiovascular disease. Current Atherosclerosis Reports, 6(5), 381-7.

Cullen, P. (2000). Evidence that triglycerides are an independent coronary heart disease risk factor. American Journal of Cardiology, 86(9), 943-949.

Devroey, D., Vantomme, K., Betz, W., Vandevoorde, J., \& Kartounian, J. (2004). A review of the treatment guidelines on the management of low levels of high-density lipoprotein cholesterol. Cardiology, 102, 61-66.

Freedman, D. S., Otvos, J. D., Jeyarajah, E. J., Shalaurova, I., Cupples, L. A., Parise, H. et al. (2004). Sex and age differences in lipoprotein subclasses measured by nuclear magnetic resonance spectroscopy: The Framingham study. Clinical Chemistry, 50(7), 1189-1200.

Garber, A. M. \& Browner, W. S. (1997). Cholesterol screening guidelines: consensus, evidence, and common sense. Circulation, 95, 1642-1645.

Garvey, T. (2003). Effects of insulin resistance and type 2 diabetes on lipoprotein subclass particle size and concentration determined by NMR. Diabetes, 52, 453-462. 
Ginsberg, H. N. (1999). Identification and treatment of hypertriglyceridemia as a risk factor for coronary heart disease. Current Cardiology Reports, 1(3), 233-240.

Gotto, A. M. (1997). The multiple risk factor intervention trial (MRFIT). A return to a landmark trial. Journal of the American Medical Association, 277, 595-597.

Grundy, S. M., Cleeman, J. I., Merz, C. N. B. et al. (2004). Implications of recent clinical trials for the national cholesterol education program adult treatment panel III guidelines. Circulation, 110, 227239.

Hertz, J. (1999). Low-density lipoprotein receptor-related protein. In D. J. Betteridge, D. R. Illingworth, \& J. Shepherd (Eds.), "Lipoproteins in health and disease (pp. 333-359)." London: Arnold.

Hsueh, W. A., \& Law, R. E. (1998). Cardiovascular risk continuum: Implications of insulin resistance and diabetes. The American Journal of Medicine, 105, 4S-14S.

Kannel, W. B., \& Wilson, P. F. W. (1992). Efficacy of lipid profiles in prediction of coronary artery disease. American Heart Journal, 124(3), 768-774.

Kannel, W., Castelli, W., Gordon, T., \& McNamara, P. (1971). Serum cholesterol, lipoproteins, and the risk of coronary heart disease: The Framingham study. Annals of Internal Medicine, 74, 1-12.

Katerndahl, D. A., \& Lawler, W. R. (1999). Variability in meta-analytic results concerning the value of cholesterol reduction in coronary heart disease: a meta-meta-analysis. American Journal of Epidemiology, 149(5), 429-441.

Kmietowicz, Z. (1998). Cholesterol screening is not worth while. British Medical Journal, 316, 725.

Kontush, A., Chantepie, S., \& Chapman, M. J. (2003). Small, dense HDL particles exert potent protection of atherogenic LDL against oxidative stress. Arteriosclerosis, Thrombosis, and Vascular Biology, 23(10), 1881-1888.

Kohlman-Trigoboff, D. (2005). Lipid management in patients with vascular disease. Journal of Vascular Nursing, 23(2), 72-726.

Kwiterovich, P. O. (1989). Beyond cholesterol. Baltimore: Johns Hopkins University Press.

Lemarche, B., Lemieux, I., \& Despres, J. P. (1999). The small, dense LDL phenotype and the risk of coronary heart disease: Epidemiology, patho-physiology and therapeutic aspects. Diabetes and Metabolism, 25, 199-211.

Malloy, M. J., \& Kane, J. P. (2001). A risk factor for atherosclerosis: Triglyceride-rich lipoproteins. Advances in Internal Medicine, 47, 111-136.

McGee, D., Reed, D., Stemmerman, G., Rhoads, G., Yano, K., \& Feinlab, M. (1985). The relationship of dietary fat and cholesterol to mortality in 10 years: The Honolulu heart program. International Journal of Epidemiology, 14, 97-105.

Muratova, V. N., Islam, S. S., Demerath, E. W., Minor, V. E., \& Neal, W. A. (2001). Cholesterol screening among children and their parents. Preventive Medicine, 33, 1-6.

National Heart, Lung, and Blood Institute. (2003). National cholesterol education program. Retrieved November 9, 2003, from http://www.nhlbi.nih.gov/about/ncep/index.htm

National Institutes of Health. (1989a). Report of the expert panel on detection, evaluation, and treatment of high blood cholesterol in adults. Washington, DC: U.S. Government Printing Office.

National Institutes of Health. (1989b). So you have high blood cholesterol. Washington, DC: U.S. Government Printing Office.

National Institutes of Health. (2002). Report of the expert panel on detection, evaluation, and treatment of high blood cholesterol in adults- third report. Washington, DC: U.S. Government Printing Office.

Ockene, I., \& Ockene, J. (1992). Prevention of coronary heart disease. Boston: Little, Brown, and Company.

Ostwald, S. K., Weiss-Farnan, P, \& Monson, T (1990). The impact of health education on health status: An experimental program for elderly women in the community. Journal of Community Health Nursing, 7(4), 175-190, 199.

Otvos, J., Jeyarajah, E., \& Cromwell, W. (2002). Measurement issues related to lipoprotein heterogeneity. American Journal of Cardiology, 90(Suppl.), 22i-29i. 
Schumaker, V., \& Lambertas, A. (1992). Lipoprotein metabolism: Chylomicrons, very-low density lipoproteins and low density lipoproteins. In A. J. Lusis, J. I. Rotter, \& R. S. Sparks (Eds.), Molecular genetics of coronary artery disease (pp. 98-139). Basel: Karger.

Smith, S. C., Jackson, R., Pearson, T. A., Fuster, Y., Faergeman et al. (2004). Principles for national and regional guidelines on cardiovascular disease prevention. A statement from the world heart and stroke forum. Circulation, 109, 3112-3121.

Stone, P. H. (2005). Evaluating cardiovascular pathophysiology and anatomy in atherosclerosis. The American Heart Hospital Journal, Summer, 3(3), 187-192.

Sullivan, D. R. (2002). Screening for cardiovascular disease with cholesterol. Clinca Chimica Acta, 315(1-2), 49-60.

Trigatti, B. L. (2005). Hepatic high-density lipoprotein receptors: roles in lipoprotein metabolism and potential for therapeutic modulation. Current Atherosclerosis Reports, Sep 7(5), 344-50

Tulenko, T. N., \& Sumner, A. E. (2002). The physiology of lipoproteins. Journal of Nuclear Cardiology: Official Publication of the American Society of Nuclear Cardiology, 9(6), 638-649.

Wada, S., \& Karino, T. (1999). Theoretical study on flow-dependent concentration polarization of low density lipoproteins at the luminal surface of a straight artery. Biorheology, 36(3), 207-223.

Wald, N. J., Law, M., Watt, H. C., Wu, T., et al. (1994). Apolipoproteins and ischemic heart disease: implications for screening. Lancet, 343, 75-79.

Wilhelmsen, L. (1997). Cardiovascular monitoring of a city over 30 years. European Heart Journal, 18, 1220-1230.

Yoshikawa, M., Sakuma, N., Hibino, T., Sato, T., \& Fujinami, T. (1997). HDL3 exerts more powerful and anti-oxidative, protective affects against copper-catalyzed LDL oxidation than HDL. Clinical Biochemistry, 30, 221-225.

Yusef, S., Hawken, S., Ounpuu, S., Dans, T., Avezum, A., Lanas, F., et al. (2004). Effect of potentially modifiable risk factors associated with myocardial infarction in 52 countries (the interheart study): Case-control study. Lancet, 364, 937.

Author Information

Rodney G. Bowden, Ph.D.*

Assistant Professor of Health Education

Health, Human Performance, and Recreation

Center for Exercise, Nutrition, \& Preventive Health Research

Baylor University

One Bear Place \# 97313

Waco, TX 76798-7313

Ph.: 254-710-4020

Fax.: 254-710-3527

E-Mail: Rodney_Bowden@baylor.edu

Stuart Hebert, BS-Honors

Research Assistant

E-Mail: stuarthebert@hotmail.com

Ronald Wilson, MD, Nephrologist

Central Texas Nephrology Associates

Waco, TX 76798

E-Mail: rbwilsonbu@hot.rr.com 
Mindy Gentile, MS, RD, LD2, Dietitian Central Texas Nephrology Associates

Waco, TX 76798

E-Mail: warren4osu@msn.com

Beth A. Lanning, Ph.D., CHES1

Assistant Professor of Health Education

E-Mail: Beth_Lanning@baylor.edu

* corresponding author 